P11.14 PHARYNGEAL AND ANOGENITAL CHLAMYDIA IN MEN WHO HAVE SEX WITH MEN: TEMPORAL TRENDS AND CHARACTERISTICS AMONG ATTENDEES AT A SYDNEY METROPOLITAN SEXUAL HEALTH CLINIC 2011-2014

${ }^{1}$ SL Goddard*, ${ }^{1} \mathrm{P}$ Rajagopal, ${ }^{1,2,3} \mathrm{DJ}$ Templeton. ${ }^{1}$ RPA Sexual Health, Sydney Local Health District, Sydney, Australia; ${ }^{2}$ The Kirby Institute, UNSW Australia, Sydney, Australia; ${ }^{3}$ Central Clinical School, University of Sydney, Sydney, Australia

\subsection{6/sextrans-2015-052270.462}

Introduction Australian STI testing guidelines recommend regular Chlamydia trachomatis (CT) testing at pharyngeal and anogenital sites among asymptomatic men who have sex with men (MSM). Our aim was to investigate temporal trends and characteristics of clinic-diagnosed pharyngeal and anogenital CT among MSM.

Methods Testing was performed using Aptima Combo 2 (Hologic, US). Data were extracted from the laboratory database and medical records. Statistical analyses were performed using STATA13 (Statcorp, College Station, TX).

Results During the study period 2919 MSM were tested on 6850 occasions, and $556(8.2 \%)$ episodes of CT were diagnosed. Pharyngeal (PCT), urethral (UCT) and rectal (RCT) positivity rates were $1.2 \%, 2.3 \%$ and $6.2 \%$, respectively. From 20112014 , there was a significant increase in number of tests performed, (999-2834, p-trend <0.001), although the positivity rate over the study period remained stable at all 3 sites. There was a non-significant decline in the proportion of those diagnosed with CT who reported any anogenital symptom during the study period $(\mathrm{OR} 0.85$, p-trend $=0.079$ ). Local symptoms were present in $24.3 \%$ and $8.0 \%$ of those with UCT and RCT, respectively. Of 85 visits where PCT was detected, almost half ( $\mathrm{n}$ $=39,45.9 \%$ ) had no concurrent anogenital infection. Of those, 2 were CT contacts and were treated at the initial visit. Men with PCT had a median of 15 sexual partners over the past year (range1-1000) which was non-significantly higher than men diagnosed with either UCT ( $p=0.077)$ or RCT $(p=0.094)$.

Conclusion CT positivity remained stable despite substantially increased testing. The temporal decline in symptomatic infections likely reflects a recent policy shift towards regular asymptomatic testing for MSM. Despite a relatively low prevalence of PCT, men with PCT had more sexual partners than men with anogenital CT and close to half the pharyngeal infections would have remained untreated without pharyngeal testing. There may be valuable public health benefits of regular PCT testing among MSM.

Disclosure of interest statement No disclosures of interest.

\section{P11.15 FACTORS ASSOCIATED WITH REPEAT SYMPTOMATIC GONORRHOEA INFECTIONS AMONG MEN WHO HAVE SEX WITH MEN, BANGKOK, THAILAND}

${ }^{1} S$ Pattanasin*, 'P Luechai, 'A Sriporn, '1 Tongtoyai, 'W Sukwicha, ${ }^{1} O$ Kongpechsatit, ${ }^{2} \mathrm{P}$ Sirivongrangson, ${ }^{1,3} \mathrm{TH}$ Holtz, ${ }^{1,3} \mathrm{ME}$ Curlin, ${ }^{1,3} \mathrm{EF}$ Dunne. ${ }^{1} \mathrm{HIV} / \mathrm{ST}$ R Research Program, Thailand Ministry of Public Health - US Centers for Disease Control and Prevention Collaboration, Nonthaburi, Thailand; ${ }^{2}$ Department of Disease Control, Ministry of Public Health, Nonthaburi, Thailand; ${ }^{3}$ Division of HIVIAIDS Prevention, US Centers for Disease Control and Prevention, Georgia, USA

\subsection{6/sextrans-2015-052270.463}

Theme C (Preventing HIV and related infections: Epidemiology and Prevention in Australia and the Region).
Background Repeat Neisseria gonorrhoeae (NG) infections indicate ongoing HIV and STI risk among men who have sex with men (MSM). We examined repeat NG among MSM enrolled in the Bangkok MSM Cohort Study (BMCS).

Methods Sexually-active Thai MSM aged $\geq 18$ years from Bangkok were enrolled in the BMCS during 2006-2008 (Period1) and 2009-2010 (Period2) and were followed every 4 months for 3-5 years. At baseline, participants were screened for rectal and urethral NG and Chlamydia trachomatis (CT) infections using a nucleic acid amplification test (NAAT). Symptomatic participants at follow-up (i.e. men with urethral or anal discharge, urethral pain and rectal pain) had urethral or rectal specimens tested for NG by NAAT, and were treated if positive. We evaluated baseline factors associated with number of symptomatic NG infections using Poisson regression with robust standard error.

Results Among 1,595 participants who had specimens at enrollment (median age 26 years, Interquartile range (IQR): 22-30 years), prevalence of rectal and urethral $\mathrm{NG}$ was $6.1 \%$ and $1.8 \%$, respectively. Of the 1,439 participants with at least one follow-up visit, 119 had NG infection at any follow-up visit. Forty-four (37.0\%) had repeat NG (range 2-7), and 21/44 $(47.7 \%)$ had only 2 infections. The median time between the first 2 infections was 294 days (IQR: 169-461 days). Factors significantly associated with number of symptomatic NG infections were enrollment in Period1 (Adjusted Incidence Rate Ratio (AIRR), 2.5, 95\% Confidence Interval (CI), 1.5-4.3), history of HIV testing without awareness of test result (AIRR 2.8, 95\% CI 1.2-6.7), history of previous STI (AIRR 2.9, 95\% CI 1.8-4.4), and prevalent CT infection (AIRR 2.2, 95\% CI 1.4-3.6).

Conclusion Repeat NG infections among BMCS participants were found and associated with other STIs. After NG diagnosis and treatment, follow-up evaluation in 3 months for repeat NG infection is warranted.

\section{P11.16 DENOMINATORS MATTER: TRENDS IN NEISSERIA GONORRHOEAE INCIDENCE AMONG GAY, BISEXUAL AND OTHER MEN WHO HAVE SEX WITH MEN (GBMSM) IN THE US - FINDINGS FROM THE STD SURVEILLANCE NETWORK (SSUN) 2010-2013}

${ }^{1} \mathrm{MR}$ Stenger*${ }^{2,3} \mathrm{H}$ Bauer, ${ }^{1} \mathrm{E}$ Torrone, ${ }^{3} \mathrm{SS}$ S Study Group. ${ }^{1}$ Division of STD Prevention, US Centers for Disease Control \& Prevention; ${ }^{2}$ STD Control Branch, California Department of Public Health, Richmond, CA; ${ }^{3} S S$ UN Working Group Includes: Greta Anschuetz, MPH (STD Control Program, Philadelphia Department of Public Health, Philadelphia, PA); Margaret Eaglin, MPH (City of Chicago Department of Public Health, Chicago, IL); Heidi Bauer (STD Control Branch, California Department of Public Health, Richmond, CA); Lynn Sosa, MD (STD Control Program, Connecticut Department of Public Health, Hartford, CT); Preeti Pathela, PhD (Bureau of STD Control and Prevention, The New York City Department of Health and Mental Hygiene, Long Island City, NY); Mary Reed, MPH (Colorado Department of Public Health and Environment, Denver, CO); Christina Schumacher, PhD (Johns Hopkins School of Medicine, Baltimore, MD); Jane Schwebke, MD (University of Alabama at Birmingham, Birmingham, AL); Julie Simon, MSPH (Infectious Disease Assessment Unit, Washington State Department of Health, Olympia, WA); Jeff Stover, MPH (Health Informatics \& Integrated Surveillance Systems, Virginia Department of Health - Division of Disease Prevention, Richmond, VA)

\subsection{6/sextrans-2015-052270.464}

Introduction Inequalities in Neisseria gonorrhoeae burden by sexual minority status have been observed in the United States but are difficult to characterise. GBMSM status of gonorrhoea cases is not routinely collected for reported cases and GBMSM population estimates at the level of geography necessary to most usefully inform public health responses are not readily available. 